\title{
A novel homozygous variant in JAM3 gene causing hemorrhagic destruction of the brain, subependymal calcification, and congenital cataracts (HDBSCC) with neonatal onset
}

\author{
Domenico Umberto De Rose ${ }^{1}$. Francesca Gallini ${ }^{2,3}$. Domenica Immacolata Battaglia ${ }^{3,4}$ - Eloisa Tiberi ${ }^{2}$. \\ Simona Gaudino ${ }^{5}$. llaria Contaldo ${ }^{4}$. Chiara Veredice ${ }^{4}$. Domenico Marco Romeo ${ }^{3,4}$. Luca Massimi ${ }^{6}$. Alessia Asaro ${ }^{7}$. \\ Cristina Cereda $^{8} \cdot$ Giovanni Vento $^{2,3} \cdot$ Eugenio Maria Mercuri $^{3,4}$
}

Received: 7 April 2021 / Accepted: 8 July 2021 / Published online: 22 July 2021

(c) Fondazione Società Italiana di Neurologia 2021

\begin{abstract}
Background JAM3 gene, located on human chromosome 11q25, encodes a member of the junctional adhesion molecule (JAM) family. Mutations of this gene are associated with hemorrhagic destruction of the brain, subependymal calcification, and congenital cataracts (HDBSCC).

Case report Herein, we present a newborn male with a prenatal suspicion of bilateral cataracts but without fetal ultrasound findings of cortical malformations. He was postnatally diagnosed with a clinical picture of HDBSCC and Early-onset Developmental and Epileptic Encephalopathy (DEE), associated to a homozygous variant of JAM3 gene.

Conclusion Identification of this variant in affected individuals has implications for perinatal and postnatal management and genetic counseling. To the best of our knowledge, this is the first case reported of a child with a JAM3 variant in Italy, from a different ethnic background than the other reported children until now (Saudi Arabian, Turkish, Afghani, and Moroccan origin). JAM3 screening could be requested in prenatal diagnosis of fetal congenital cataracts and included in NextGeneration DNA Sequencing panels.
\end{abstract}

Keyword JAM3; Seizures; Cerebral hemorrhages; Cataracts; Neonate; Infant; COL4A1

\section{Background}

JAM3, located on human chromosome $11 \mathrm{q} 25$, is a gene that encodes a member of the junctional adhesion molecule (JAM) family [1-3].

Domenico Umberto De Rose

derosedomenicoumberto@gmail.com

1 Neonatal Intensive Care Unit, Medical and Surgical Department of Fetus - Newborn - Infant, "Bambino Gesù” Children's Hospital IRCCS, Piazza S. Onofrio 4, 00165 Rome, Italy

2 Neonatology Unit, Department of Woman and Child Health and Public Health, Fondazione Policlinico Universitario "Agostino Gemelli” IRCCS, Rome, Italy

3 Università Cattolica del Sacro Cuore, Rome, Italy

4 Pediatric Neurology Unit, Department of Woman and Child Health and Public Health, Fondazione Policlinico Universitario "Agostino Gemelli” IRCCS, Rome, Italy
Mutations of this gene are associated with hemorrhagic destruction of the brain, subependymal calcification, and congenital cataracts (HDBSCC, OMIM \#613,730) [4, 5].

By genome wide linkage analysis followed by candidate gene sequencing of a Saudi Arabian family with HDBSCC, Mochida et al. identified a homozygous loss-of-function

5 Radiology Unit, Department of Diagnostic Imaging, Radiotherapy, Oncology and Hematology, Fondazione Policlinico Universitario “Agostino Gemelli” IRCCS, Rome, Italy

6 Pediatric Neurosurgery Unit, Fondazione Policlinico Universitario “Agostino Gemelli” IRCCS, Rome, Italy

7 Genomic and Post-Genomic Center, IRCCS Mondino Foundation, Pavia, Italy

8 Neonatal Screening and Metabolic Disorders Unit, Department of Woman, Mother and Neonate, "V. Buzzi" Children's Hospital - ASST Fatebenefratelli Sacco, Milan, Italy 
mutation in the JAM3 gene (OMIM*606,871). Affected individuals either died in early infancy or survived with profound developmental delay, spasticity, and seizures [4]. These findings showed how much JAM3 is important for maintaining the integrity of cerebral endothelial cells (avoiding hemorrhages) and for normal lens development in humans (avoiding cataract), confirming data seen in JAM3deficient mice [6-8].

Three years later, in affected members of 3 unrelated families with HDBSCC (one Turkish, one Afghani, and one Moroccan), Akawi et al. identified 3 different homozygous loss-of-function mutations in JAM3 gene [5], with a similar phenotype to that reported by Mochida in 2010 [4].

Herein, we present a newborn male with a prenatal suspicion of bilateral cataracts but without fetal ultrasound findings of cortical malformations. He was diagnosed postnatally with a clinical picture of HDBSCC associated to a mutation of JAM3 gene: the 24-month follow-up was described.

\section{Results}

\section{Clinical report}

An Italian child was born to healthy but consanguineous parents at 37 weeks of gestational age (GA). Both parents were from central Italy. His 36-year-old mother had no symptomatic infections and was negative on TORCH infection screening during pregnancy. He was the first child and had no family history of neurological disorders or inherited cataracts. Bilateral crystalline hyperechogenities were previously detected at ultrasound assessment of 20 weeks GA. However, fetal magnetic resonance imaging (MRI) excluded a cataract and ischemic-hemorrhagic cerebral abnormalities at 20 weeks and 5 days GA.

The baby was delivered at term via a normal spontaneous delivery, with an Apgar score of 9 and 10 at 1 and 5 min after birth, respectively. The child's auxological parameters at birth were all appropriate for gestational age according INeS Charts: weight $2750 \mathrm{~g}$ ( $25^{\text {th }}$ centile), length $48 \mathrm{~cm}$ $\left(25-50^{\text {th }}\right.$ centile); head circumference $34 \mathrm{~cm}\left(50^{\text {th }}\right.$ centile $)$. No major malformations were detected, except for a bilateral cataract. At about $12 \mathrm{~h}$ of life, he was irritable and exhibited excessive startle, hypotonia, and apnea. Therefore, he was transferred from maternity room to our Neonatal Intensive Care Unit (NICU), where a cranial ultrasound showed periventricular leukomalacia.

Focal seizures started in second day of life, presenting with non-motor/autonomic signs (cyanosis and tachycardia) followed by bilateral myoclonic jerks and generalized hypertonia. Electroclinical and electrographic only episodes were observed. Seizures became more and more frequent until status epilepticus that was refractory to phenobarbital; the infant required mechanical ventilation. The status epilepticus was controlled with continuous infusion of midazolam. An Early-onset Developmental and Epileptic Encephalopathy (DEE) was documented. Multiple intraventricular hemorrhages (IVH) and intraparenchymal hemorrhages (IPH) were detected on brain magnetic resonance imaging (MRI): hemorrhages were observed, in particular, in left hemisphere (frontal and temporo-occipital regions), without midline shift. Other abnormalities included multiple periventricular calcifications, porencephalic cysts, and hypoplasia of cerebellar vermis (Fig. 1). The progressive post-hemorrhagic hydrocephalus (PHH) was managed with a ventriculo-subgaleal shunt (VSGS) on $12^{\text {th }}$ day of life.

An extensive metabolic examination for plasma and urinary amino acids and urinary organic acids, and screening for galactosemia were all normal. Creatine kinase (CK) concentration was normal. Initially a TORCH infection was suspected, but all tests for toxoplasmosis, rubella, cytomegalovirus, and herpes simplex virus were negative for the child and her mother. Therefore, we hypothesized that the patient had an underlying genetic disease that was associated with vascular vulnerability. Array-comparative genomic hybridization (array-CGH) was ordered and did not disclose any alterations.

A complete heart assessment revealed a myocardial hypertrophy. Subclinical arterial hypertension was noticed at 1 month of age, and the presence of renal hypertension was identified based on the elevation of the plasma renin activity; however, abdomen ultrasonography examination detected no internal organ defects, and no apparent stenosis was detected in either of the renal arteries. An oral calcium channel blocker (amlodipine $0.2 \mathrm{mg} / \mathrm{kg} /$ day) was transiently administered with a good control of blood pressure.

Ophthalmological examination showed congenital bilateral cataract, without other abnormalities. Vitrectomy was performed at 2 months of life.

A good seizure control was gradually obtained using levetiracetam and phenobarbital. The baby was then discharged from NICU with a strict follow-up program.

\section{Genetic analysis}

Considering suspected cortical malformations and cataract, the coding regions of $C O L 4 A 1$ and JAM 3 genes were analyzed by performing a Next Generation Sequencing by Nextera (Illumina) assay for targeted resequencing. After bioinformatics analysis and variant filtering and prioritization, the patient was found to carry a novel homozygous variant in exon 6 of JAM3 gene: c.690 T > G (NM_032801.5) p.Cys230Trp (NP_116190.3). This homozygous variant was confirmed by Sanger sequencing. His father and his mother were both heterozygous carriers 

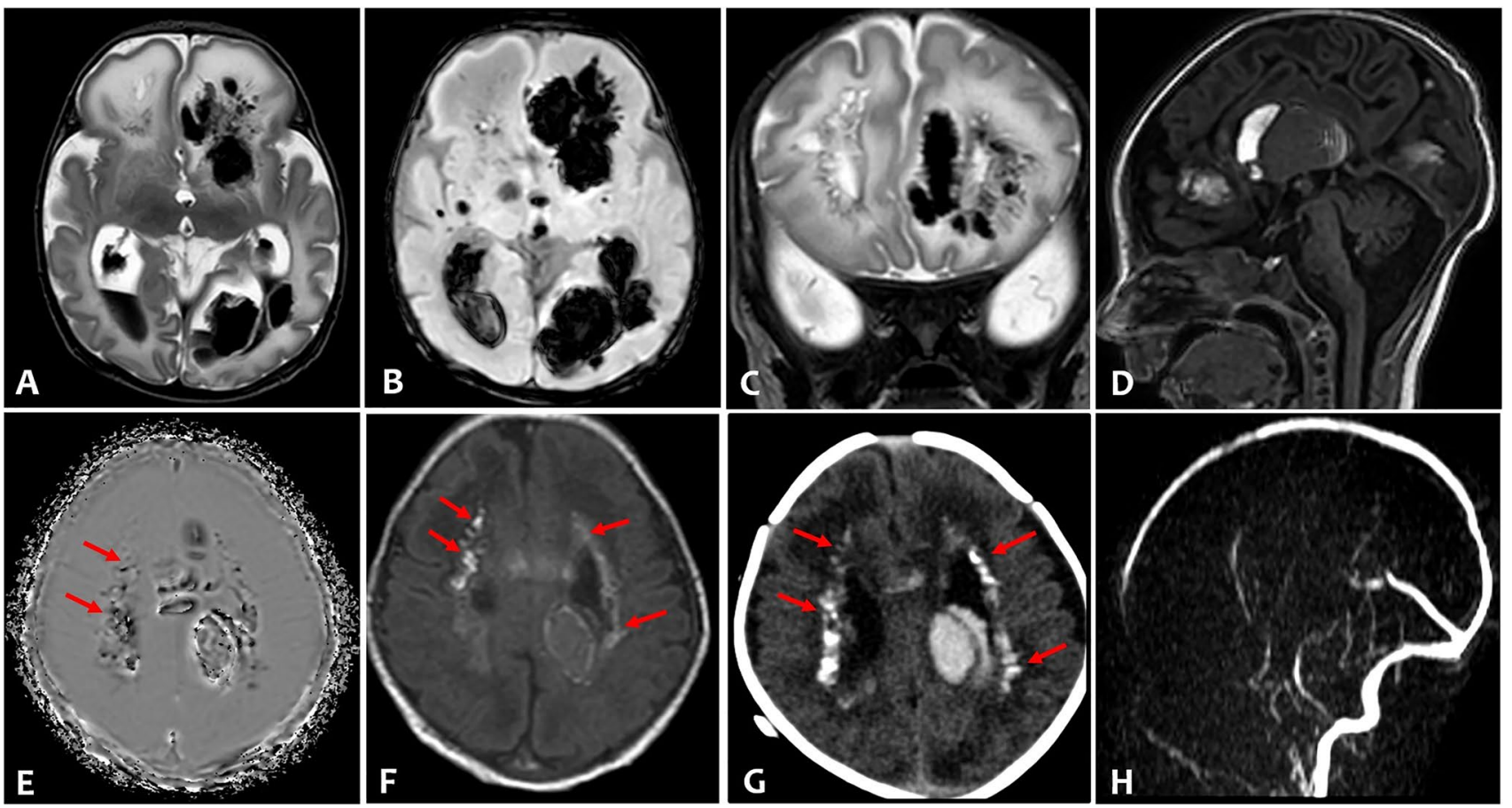

Fig. 1 Axial T2-weighted (A) and susceptibility weighted imaging (B) of brain MRI showed severe intraventricular hemorrhage and bilateral multiple intraparenchymal hemorrhages, prevailing in the left hemisphere, and enlargement of the posterior horns of the lateral ventricles. Coronal T2-weighted (C) showed multiple cysts in the frontal periventricular white matter. Sagittal T1-weighted (D) revealed also a large intraventricular clot in the III ventricle and hypo-

plasia of cerebellar vermis. Multiple periventricular calcifications (arrows) were demonstrated on phase axial susceptibility weighted image (E), on T1-weighted (F) image, and on corresponding axial CT (G). There was no evidence of venous thrombosis and vascular malformation on MR venogram $(\mathbf{H})$ and time of flight angiography (not shown)

Fig. 2 (A) Sanger sequencing confirmed the homozygous variation, c.690 T > G, in the proband. (B and $\mathbf{C}$ ) Sanger sequencing shown that the parents were both carrying the heterozygous variation

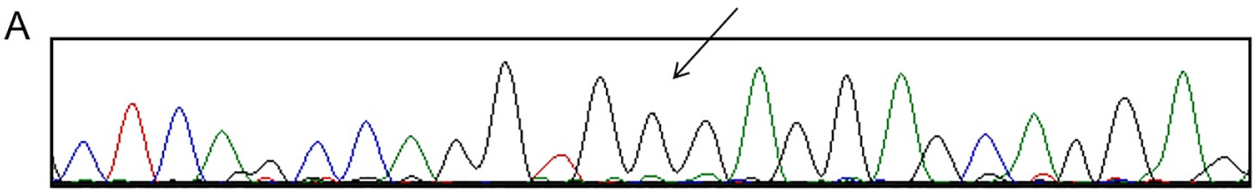

B

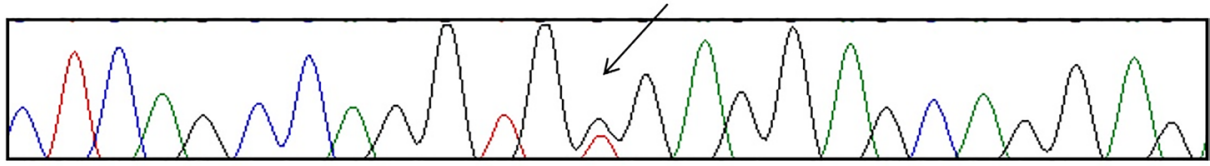

C

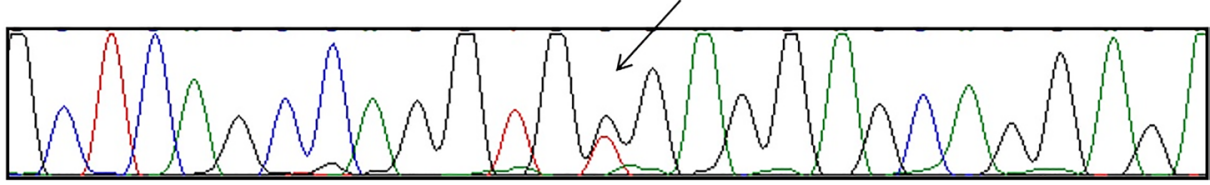

(Fig. 2). JAM3 mutations have been reported as associated to recessive forms of brain hemorrhages, subependymal calcifications and cataract (OMIM \#606,871). This variant has not been reported until now in literature and is highly likely to be pathogenic according to American College of Medical Genetics (ACMG) criteria [9]. We also assessed the pathogenicity of this mutation using Poly-Phen2 software (http://genetics.bwh.harvard.edu/pph2/index.shtml) that identified it as "Probably Damaging", suggesting that this novel variant has high pathogenicity. Identified variant was classified according to the HGVS nomenclature (www.hgvs.org/mutnomen). 


\section{Follow-up}

During a 2-year follow-up (Fig. 3A-D), he showed a marked growth impairment; an Early-Onset Developmental and Epileptic Encephalopathy (DEE) was confirmed, associated with spastic tetraparesis. He received percutaneous endoscopic gastrostomy (PEG) for feeding.

Serial head ultrasound and brain MRI showed a gradual worsening of ventricular dilatation (Fig. 4A,F; B,G): a ventriculoperitoneal shunt (VPS) was implanted at 4 months of life. Subsequent MRI (Fig. 4C, H; D,I) showed enlargement
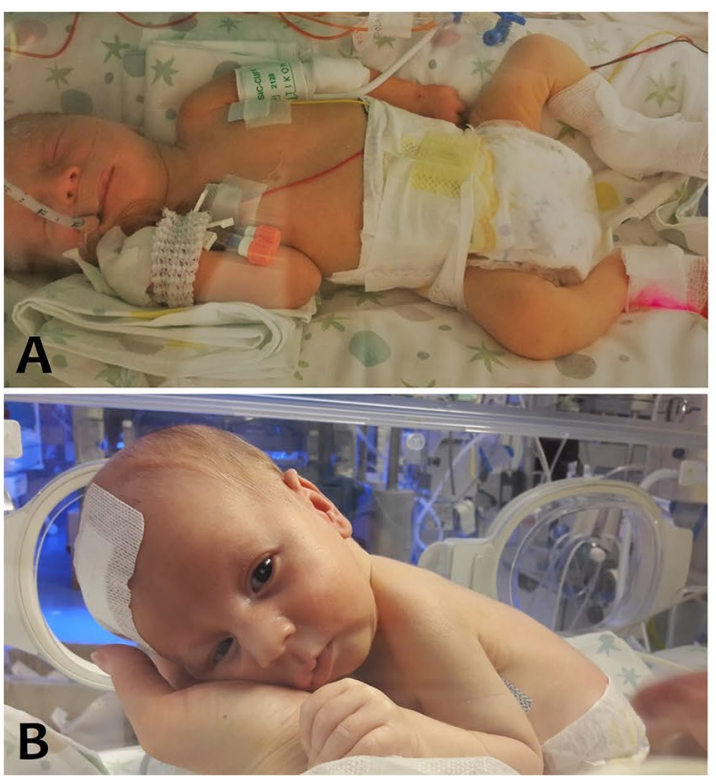
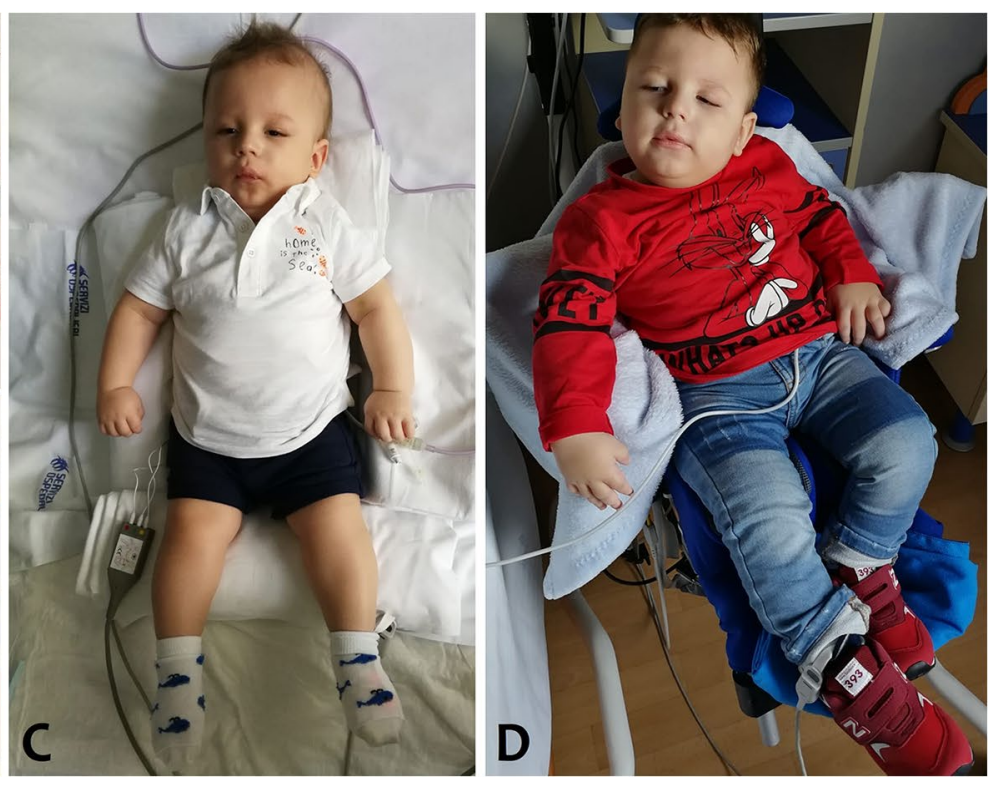

Fig. 3 The patient during NICU stay (A-B), at 12 months (C) and at 24 months (D)

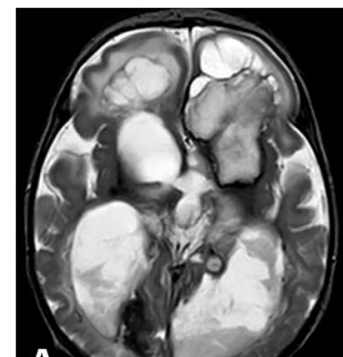

A

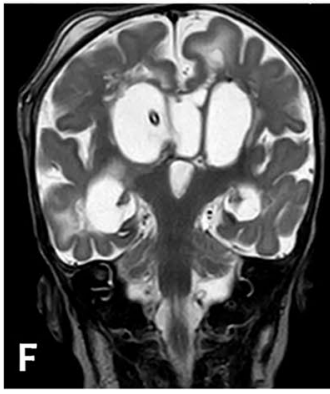

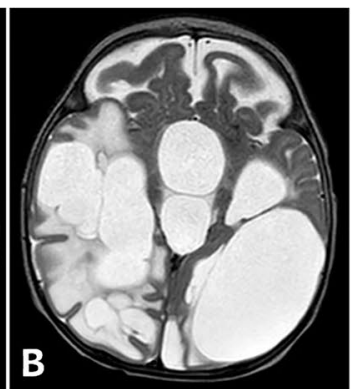
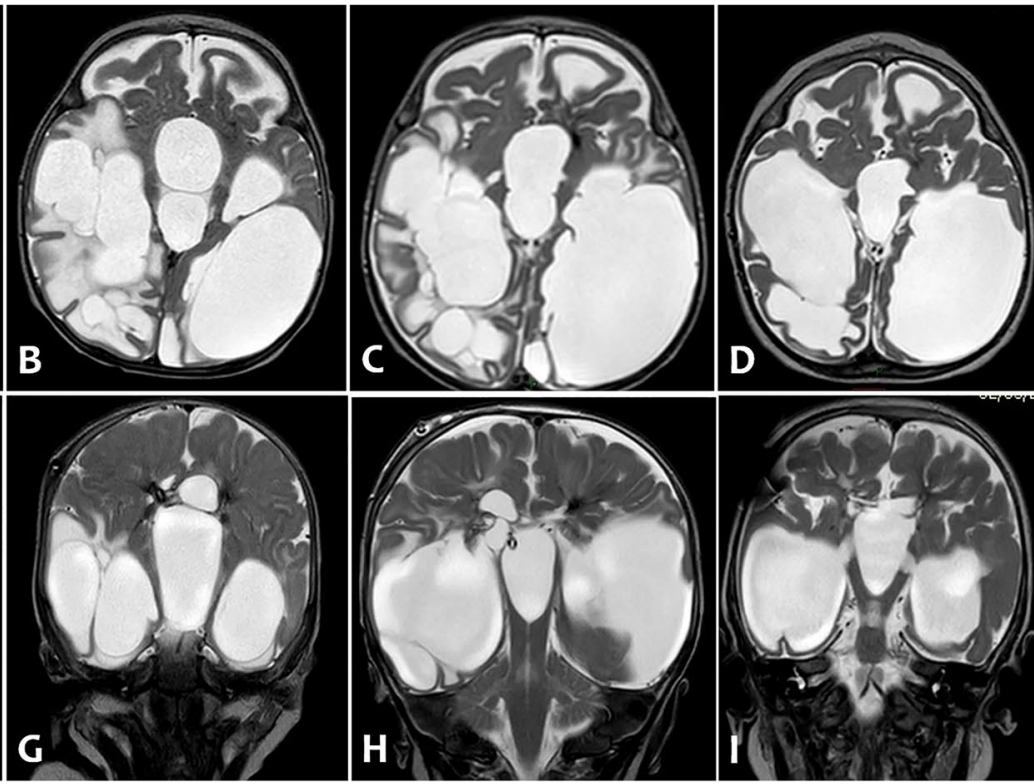
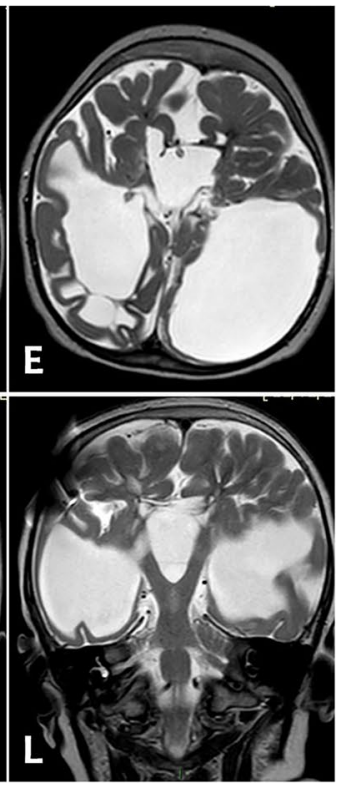

Fig. 4 Axial (A-E) and coronal (F-L) T2-weighted images of serial brain MRI: (A,F) 1 month of life, $(\mathbf{B}-\mathbf{G}) 3$ months of life, $(\mathbf{C}-\mathbf{H})$ 4 months of life, (D-I) 15 months of life, (E-L) 2 years of life. Serial MRI scans showed porencephalic cystic evolution and reduced whitematter volume (indicative of destructive changes), and consensual gradual worsening of ventricular dilatation unless a ventriculoperitoneal shunt was implanted at 4 months of life. Last two MRI exams (D-I and E-L) showed a plastic re-organization of porencephalic cysts and lateral ventricles with reduction of signs of cerebrospinal fluid's decompensation 
of porencephalic cystic and reduction of white-matter volume (destructive changes), and consensual gradual worsening of ventricular dilatation. At 2 years of life, brain MRI showed a plastic re-organization of porencephalic cysts and lateral ventricles with reduction of signs of cerebrospinal fluid's decompensation (Fig. 4E,L).

The epileptic disorder was characterized by focal drugresistant epilepsy, requiring to different antiepileptic drugs (AEDs) be added/substituted. Awake and sleep electroencephalogram (EEG) studies showed a severe alteration of brain electrical activity due to the organization of the background rhythm (a diffuse theta rhythm with posterior predominance) and due to the presence of intermittent bursts of high-voltage sharp waves and spikes. At time of writing, he receives topiramate, clonazepam, vigabatrin, lamotrigine with a sufficient seizure control.

Microphthalmia is evident; ocular motility is poorly evaluable, with horizontal and rotary nystagmus jerks. Spontaneous mobility is reduced with intermittent occurrences of abrupt and synchronized movements of the limbs. He shows a spastic quadriplegia with axial hypotonia. He has no head control and no functional use of the hands. A spinal X-ray performed at two years showed a dorsal-lumbar scoliosis. He wears ankle-foot orthosis, and he sits on a polyfunctional chair.

During the Italian COVID-19 s wave, he was found positive for SARS-CoV-2 by real-time PCR on nasopharyngeal swab. However, he is still alive and never developed fever or any signs of infection.

\section{Discussion}

Dreadful cerebrovascular diseases could be related to impaired integrity of blood vessels, as we describe in this clinical report of a term neonate with early-onset multiple intraventricular hemorrhages (IVH) and intraparenchymal hemorrhages (IPH).

Recent studies have tested the hypothesis that IVH may be secondary to variability in some risk genes [10]. Among these genes, COL4A1 gene (OMIM*120,130), located on human chromosome 13q34, encodes type IV collagen alpha- 1 chain protein, which is expressed in all human tissues, especially in vessels and brain. To date, many pathogenic COLAA1 mutations have been identified. A wide spectrum of clinical manifestations, associated with $C O L 4 A 1$ mutations, may be caused by aberration of basement membranes of blood vessels. Neurological features are the most prominent but are rather nonspecific: antenatal intracranial hemorrhage, porencephaly, brain small-vessel disease with hemorrhage or vascular leukoencephalopathy have been reported [11-17]. Ophthalmological features associated with
COL4A1 mutations are heterogeneous and include congenital cataracts [18].

Similarly, dysfunction of the tight junction components (occludin - OCLN, and junctional adhesion molecules JAMs) has been associated with an overlap clinical presentation. There are three types of JAMs: JAMI (expressed in endothelial and epithelial cells) and JAM2 and JAM3 (expressed only in vascular endothelial cells). JAM-1 is a ligand involved in trans-endothelial migration of leukocytes [19]. JAM2 plays a key role in blood-brain-barrier permeability and bi-allelic JAM2 variants have been related to early-onset recessive primary familial brain calcification (OMIM \#618,824): Schottlaender et al. suggested that defective cell-to-cell adhesion and dysfunction of the movement of solutes through the paracellular spaces in the neurovascular unit could be a key mechanism in central nervous system calcifications [20]. JAM-3 functions similarly to JAM-2 and its mutations have been identified to cause hemorrhagic destruction of the brain [4, 5]. According to findings by Daniele et al. (2007), mouse JAM3 apical expression was also found on the embryonic retinal neuroepithelia, and its deficiency caused nuclear cataracts [21].

Conversely, OCLN is associated with autosomal recessive Pseudo-TORCH syndrome 1 (OMIM \#251,290): affected individuals have congenital microcephaly, intracranial calcifications, simplified gyration and polymicrogyria, and severe developmental delay [22]. Patients with OCLN mutations did not have intracranial hemorrhages or congenital cataracts; only two siblings reported by Slee et al. (1999) also presented with congenital cataracts [23].

Herein, we reported a novel homozygous variant in the tight-junction gene JAM3 in an Italian child with severe hemorrhagic destruction of the brain and bilateral congenital cataracts as cardinal features. He was diagnosed postnatally with a clinical picture of HDBSCC. Echocardiography displayed no anatomical heart defects, but only myocardial hypertrophy. We did not observe in our patient thrombocytopenia, hepatomegaly, renal anomalies, or other organ involvement as previously reported in some patients $[4,5]$. As reported by Akawi et al., the disorder is likely due to the total loss of the protein function as a result of the mutation [5].

COL4A1-related disease could have an overlap clinical presentation: according Tonduti et al., raised CK concentration, in addition to intracranial calcifications, is to be considered a useful pointer to a final diagnosis of COL4A1-related disease [24].

Our clinical report stresses the importance of screening also any JAM3 mutation when CK concentration is normal, such as in our patient. A normal CK concentration, in addition to intracranial calcifications and diffuse cerebral hemorrhages, should therefore lead to analyze JAM3 gene in the suspicion of HDBSCC. 
To the best of our knowledge, this is the first case reported of a child with a JAM3 variant in Italy, from a different ethnic background than the other reported children until now (Saudi Arabian, Turkish, Afghani, and Moroccan origin).

Furthermore, congenital cataract is a genetically heterogeneous disease [25]. Next-generation DNA sequencing (NGS) technologies have been validated to determine the precise genetic cause of bilateral congenital cataracts in about $75 \%$ of individuals but JAM3 is not usually included in these panels $[26,27]$. Our results contribute to expand the mutational spectrum of genes causing congenital cataract, in approaching the prenatal diagnosis.

\section{Conclusion}

In conclusion, we reported a phenotype resembling a TORCH infection caused by a novel variant in JAM3. We suggest COLAAI and JAM 3 testing in patients with posthemorrhagic hydrocephalus, porencephalic cysts, and cataract as well as suspected TORCH infection without any proven etiological factors. Furthermore, JAM3 screening could be requested in prenatal diagnostic work-up of fetal congenital cataracts and included in NGS panels.

This is important, as identification of this variant in affected individuals has implications for perinatal and postnatal management and genetic counseling. In addition, making this diagnosis may help tailor appropriate screening tests for organs typically catastrophically involved.

\section{Conflict of interest}

The authors declare no potential conflict of interest with respect to the research, authorship and/or publication of this article.

\section{Ethical approval}

The authors declare that they have followed the protocols of their Institutional Review Board on the publication of the patient's data and the Helsinki Declaration; no patient is recognizable from data within this article. We obtained written informed consent from the parents beforehand in order to report these findings.

Acknowledgements The authors thank the parents for their kindness and gratitude toward us.

\section{References}

1. Arrate MP, Rodriguez JM, Tran TT et al (2001) Cloning of human junctional adhesion molecule 3 (JAM3) and its identification as the JAM2 counter-receptor. J Biol Chem 276:45826-45832

2. Liang TW, Chiu HH, Gurney A et al (2002) Vascular endothelialjunctional adhesion molecule (VE-JAM)/JAM 2 interacts with T, NK, and dendritic cells through JAM 3. J Immun 168:1618-1626

3. Santoso S, Sachs UJH, Kroll H et al (2002) The junctional adhesion molecule 3 (JAM-3) on human platelets is a counterreceptor for the leukocyte integrin Mac-1. J Exp Med 196:679-691

4. Mochida GH, Ganesh VS, Felie JM et al (2010) A homozygous mutation in the tight-junction protein JAM3 causes hemorrhagic destruction of the brain, subependymal calcification, and congenital cataracts. Am J Hum Genet 87:882-889

5. Akawi NA, Canpolat FE, White SM et al (2013) Delineation of the clinical, molecular and cellular aspects of novel JAM3 mutations underlying the autosomal recessive hemorrhagic destruction of the brain, subependymal calcification, and congenital cataracts. Hum Mutat 34:498-505

6. Gliki G, Ebnet K, Aurrand-Lions M et al (2004) Spermatid differentiation requires the assembly of a cell polarity complex downstream of junctional adhesion molecule-C. Nature 431:320-324

7. Imhof BA, Zimmerli C, Gliki G et al (2007) Pulmonary dysfunction and impaired granulocyte homeostasis result in poor survival of Jam-C-deficient mice. J Pathol 21:198-208

8. Daniele LL, Adams RH, Durante DE et al (2007) Novel distribution of junctional adhesion molecule-C in the neural retina and retinal pigment epithelium. J Comp Neurol 505:166-176

9. Richards S, Aziz N, Bale S et al (2015) Standards and guidelines for the interpretation of sequence variants: a joint consensus recommendation of the American College of Medical Genetics and Genomics and the Association for Molecular Pathology. Genet Med 17(5):405-424

10. Adén U, Lin A, Carlo W et al (2013) Candidate gene analysis: severe intraventricular hemorrhage in inborn preterm neonates. J Pediatr 163(5):1503-6.e1

11. Smigiel R, Cabala M, Jakubiak A et al (2016) Novel COL4A1 mutation in an infant with severe dysmorphic syndrome with schizencephaly, periventricular calcifications, and cataract resembling congenital infection. Birth Defects Res A Clin Mol Teratol 106(4):304-307

12. Lichtenbelt KD, Pistorius LR, De Tollenaer SM et al (2012) Prenatal genetic confirmation of a COL4A1 mutation presenting with sonographic fetal intracranial hemorrhage. Ultrasound Obstet Gynecol 39(6):726-727

13. Livingston J, Doherty D, Orcesi S et al (2011) COL4A1 mutations associated with a characteristic pattern of intracranial calcification. Neuropediatrics 42(6):227-233

14. Vermeulen RJ, Peeters-Scholte C, Van Vugt JJ et al (2011) Fetal origin of brain damage in 2 infants with a COL4A 1 mutation: fetal and neonatal MRI. Neuropediatrics 42(1):1-3

15. Meuwissen ME, de Vries LS, Verbeek HA et al (2011) Sporadic COL4A1 mutations with extensive prenatal porencephaly resembling hydranencephaly. Neurology 76(9):844-846

16. Bilguvar K, DiLuna ML, Bizzarro MJ et al (2009) COL4A1 mutation in preterm intraventricular hemorrhage. J Pediatr 155(5):743-745

17. de Vries LS, Koopman C, Groenendaal F et al (2009) COL4A1 mutation in two preterm siblings with antenatal onset of parenchymal hemorrhage. Ann Neurol 65(1):12-18

18. Coupry I, Sibon I, Mortemousque B et al (2010) Ophthalmological features associated with COL4A1 mutations. Arch Ophthalmol 128(4):483-489 
19. Ostermann G, Weber KS, Zernecke A et al (2002) JAM-1 is a ligand of the beta(2) integrin LFA-1 involved in transendothelial migration of leukocytes. Nat Immunol 3(2):151-158

20. Schottlaender LV, Abeti R, Jaunmuktane Z et al (2020) Bi-allelic JAM2 variants lead to early-onset recessive primary familial brain calcification. Am J Hum Genet 106(3):412-421

21. Daniele LL, Adams RH, Durante DE et al (2007) Novel distribution of junctional adhesion molecule-C in the neural retina and retinal pigment epithelium. J Comp Neurol 505(2):166-176

22. O'Driscoll MC, Daly SB, Urquhart JE et al (2010) Recessive mutations in the gene encoding the tight junction protein occludin cause band-like calcification with simplified gyration and polymicrogyria. Am J Hum Genet 87(3):354-364

23. Slee J, Lam G, Walpole I (1999) Syndrome of microcephaly, microphthalmia, cataracts, and intracranial calcification. Am J Med Genet 84(4):330-333
24. Tonduti D, Pichiecchio A, La Piana R et al (2012) COL4A1related disease: raised creatine kinase and cerebral calcification as useful pointers. Neuropediatrics 43(5):283-288

25. Deng H, Yuan L (2014) Molecular genetics of congenital nuclear cataract. Eur J Med Genet 57(2-3):113-122

26. Gillespie RL, O'Sullivan J, Ashworth J et al (2014) Personalized diagnosis and management of congenital cataract by nextgeneration sequencing. Ophthalmol 121(11):2124-37.e1-2

27. Astiazarán MC, García-Montaño LA, Sánchez-Moreno F et al (2018) Next generation sequencing-based molecular diagnosis in familial congenital cataract expands the mutational spectrum in known congenital cataract genes. Am J Med Genet A 176(12):2637-2645

Publisher's Note Springer Nature remains neutral with regard to jurisdictional claims in published maps and institutional affiliations. 